\title{
La soledad y el aislamiento social podrian asociarse con mayor riesgo de enfermedad cardio y cerebrovascular
}

Loneliness and social isolation could be associated to a greater risk of cardiovascular and cerebrovascular disease

Valtorta NK, y col. BMJ 2016;102:1009-16.

\section{Objetivos}

Evaluar la asociación entre la soledad y/o el aislamiento social y la incidencia de enfermedad cardiovascular (ECV) y/o accidente cerebrovascular (ACV), y si ésta varía según edad, género, estado civil, posición socio-económica, etnia o estado de salud.

\section{Fuentes de datos}

Se realizaron búsquedas en 16 bases de datos electrónicas para la literatura publicada y gris hasta mayo de 2015: MEDLINE, EMBASE, CINAHL Plus, PsycINFO, ASSIA, Web of Science, Cochrane Library, Social Policy and Practice, ETHOS, NDLTD, NHS Evidence, SCIE y el Instituto Nacional de Excelencia en Salud y Atención (NICE). Además se buscaron citas de referencias en SCOPUS y se contactaron expertos en el tema que trabajaban en el centro de Investigación de la campaña para terminar con el aislamiento social en Reino Unido.

\section{Selección de estudios}

Estudios observacionales longitudinales de países de altos ingresos que evaluaran incidencia de ECV y/o ACV según nivel de soledad y/o aislamiento social. Estos estudios debían definir ECV y ACV con los criterios diagnósticos del CIE-10, soledad como la percepción negativa de los vínculos sociales y aislamiento social como una medida objetiva de ausencia de relaciones, lazos o contacto con otros. Quedaron excluidos los estudios en los que la ECV y/o el ACV no eran los principales diagnósticos de las poblaciones.

Dos investigadores independientes seleccionaron los estudios siguiendo un formulario de cribado. Si era necesario, se solicitó información a los autores o se buscó en publicaciones relacionadas.

\section{Extracción de datos}

Los datos fueron extraídos de forma estandarizada por un investigador y comprobados por un segundo. De haber datos faltantes en algún estudio, se contactaban con los autores de los estudios primarios.

\section{Resultados principales}

Se incluyeron 23 estudios. Los pacientes con soledad y/o aislamiento social presentaron mayor riesgo relativo de ECV y ACV (véase Tabla 1).

Tabla 1: Riesgo relativo de enfermedad cardiovascular y accidente cerebrovascular en pacientes con soledad y/o aislamiento social.

\begin{tabular}{c|c}
\hline Condición & RR (IC 95\%) \\
\hline Enfermedad Cardiovascular & $1,29(1,04$ a 1,59$)$ \\
\hline Accidente Cerebrovascular & $1,32(1,04$ a 1,68) \\
\hline
\end{tabular}

RR: riesgo relativo, IC: intervalo de confianza

\section{Conclusiones}

La soledad y/o el aislamiento social, se asociaron con un aumento del $29 \%$ del riesgo de ECV, y del $32 \%$ de ACV. No se pudo evaluar si esta asociación variaba según edad, sexo, posición socio-económica y otras variables, dado los pocos datos aportados por los estudios.

Fuente de financiamiento/Conflictos de interés de los autores: los autores de este trabajo reportaron no poseer conflictos de interés.

\section{Comentario}

La soledad se define como la percepción negativa de los vínculos sociales, y el aislamiento social como una medida objetiva de ausencia de relaciones, lazos o contacto con otras personas. En la actualidad existe evidencia que posiciona a estas dos condiciones como factores de riesgo de enfermedad cardiovascular comparables a otros ya bien conocidos, tales como el tabaquismo, la hipertensión arterial, la obesidad y el sedentarismo ${ }^{1,2}$. Este trabajo estimó el riesgo de ECV y ACV de aproximadamente el $30 \%$ en esta población. Si bien muchos estudios incluidos en este meta-análisis fueron de baja calidad, mostraron moderada heterogeneidad y la mitad presentó un ajuste por confundidores inadecuado, igualmente podría inferirse que hay riesgo asociado dado que los análisis de sensibilidad excluyendo los estudios de mayor riesgo de sesgo mostraban asociaciones más fuertes. Aunque asociación no implica causalidad, abordar la soledad y el aislamiento social podría tener un papel importante en la prevención de dos de las principales causas de morbimortalidad de los países de altos ingresos, lo cual justificaría estudios experimentales para definirlo.

\section{Conclusiones del comentador}

La soledad y el aislamiento social son hoy en día un tema de preocupación entre la comunidad médica, siendo un problema en sí mismo, más allá de los riesgos en ECV y ACV. La pregunta que nos podríamos plantear sería: si la soledad y el aislamiento social son un problema en sí mismo, ¿Por qué no abordarlo desde la salud pública independientemente de las consecuencias cardio o cerebrovasculares?

Camila Volij [ Servicio de Medicina Familiar y Comunitaria del Hospital Italiano de Buenos Aires camila.volij@ hospitalitaliano.org.ar ]

Volij C. La soledad y el aislamiento social podrían asociarse con mayor riesgo de enfermedad cardio y cerebrovascular. Evid Actual Pract Ambul 2018;21 (3):88. Comentado de: Valtorta NK, y col. Loneliness and social isolation as risk factors for coronary heart disease and stroke: systematic review and meta-analysis of longitudinal observational studies. Heart 2016;102:1009-16. PMID: 27091846.

\section{Referencias}

1. Pantell M y col. Social isolation: a predictor of mortality comparable to traditional clinical risk factors. Am J Public Health 2013:103:2056-62.

2. Valtorta. Loneliness, social isolation and social relationships: what are we measuring? A novel framework for classifying and comparing tools. BMJ open 2016 vol: 6 (4) pp: e010799 